\title{
Qualitätsverbesserung bei Polytraumatisierten
}

\begin{abstract}
Ehebliche Fortschritte in der Behandlung der Polytraumatisierten haben in den letzten 25 Jahren dazu beigetragen, daß die Letalität von früher über $40 \%$ auf unter $20 \%$ bei vergleichbaren Verletzungsmustern gesunken ist. Diese Erfolge sind auf verbesserte präklinische Maßnahmen ebenso wie auf Fortschritte in der Schockforschung, der Polytraumaevaluation sowie auf operationstechnische und intensivmedizinische Innovationen zurückzuführen. Der Mangel an effektiven und validen Daten hat international in vielen Ländern die Einrichtung von Traumazentren über lange Jahre verzögert. Verschiedene groß angelegte Studien, speziell die MTOS-Stu-
\end{abstract}

dien in den USA und England, haben Wege zur Qualitätsverbesserung beim Polytraumatisierten eröffnet. Keine Berücksichtigung finden in diesen Studien - mit Ausnahme des Deutschen Traumaregisters - therapeutische Maßnahmen, die Lebensqualität und häufig auch die posttraumatische Morbidität. Der Begriff Lebensqualität wurde erstmals 1977 als separate Überschrift im Index Medicus aufgeführt. Die Beurteilung der Lebensqualität ist individuell sehr verschieden. Sie hängt u. a. von der Persönlichkeitsstruktur, dem Kulturkreis und der persönlichen Lebenserfahrung des Patienten ab. Aus dieser Individualität entsteht das Problem der Quantifizierung der Lebensqualität. Die Messung der Lebensqualität sollte nach internationaler Übereinstimmung körperliche, psychische, soziale und symptombeschreibende Komponenten mit einbeziehen. Bei allen Erfolgen, die wir mit der Senkung der Todeszahlen beim Polytrauma erreicht haben, darf dieses Problem der physischen, psychischen und sozialen Rehabilitation - gerade der Schwerverletzten - nicht aus den Augen gelassen werden. Auch dies ist ein sehr wichtiger Teil unserer unfallchirurgischen Aufgaben.

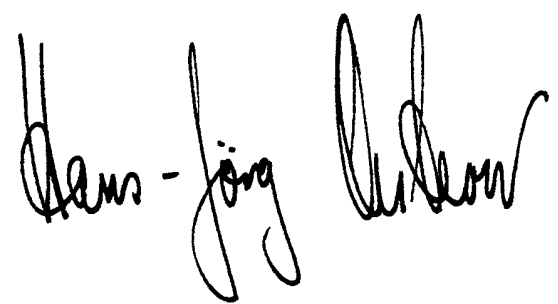

Prof. Dr. H.-J. Oestern 